
\title{
Wet Etching and Surface Analysis of Chemically Treated InGaN
} Films

\author{
N. Karar, ${ }^{\text {a,c,z }}$ R. Opila, ${ }^{\text {a }}$ and T. Beebe, Jr. ${ }^{\text {b }}$ \\ ${ }^{a}$ Department of Materials Science and Engineering and ${ }^{b}$ Department of Chemistry and Biochemistry, University of Del- \\ aware, Newark, Delaware 19716, USA
}

This paper discusses the performance of different wet chemical etchants on InGaN. It is shown that certain etchants can be used to chemically etch and remove appreciable amounts of InGaN even though the etch rate is not as high as observed for other III-V materials. The performance of etchants studied here were (i) two different ratios of $\mathrm{HF}, \mathrm{HNO}_{3}$, (ii) cyclic usage of $\mathrm{NH}_{4} \mathrm{OH}$ followed by $\mathrm{HCl}$, (iii) hot $\mathrm{H}_{2} \mathrm{SO}_{4}$ and $\mathrm{H}_{3} \mathrm{PO}_{4}$ mixture, and (iv) conc. $\mathrm{NH}_{4} \mathrm{OH}$. The etched surfaces have then been analyzed by $\mathrm{x}$-ray photoelectron spectroscopy (XPS). Different etch residues were observed on the top surface. These results suggest an alternative to reactive plasma etching or photo-enhanced electrochemical etching of InGaN type materials. Based on the observed performance of the etchants studied, it was also possible to segregate the surface cleaning protocols and etchants.

(C) 2011 The Electrochemical Society. [DOI: 10.1149/1.3574036] All rights reserved.

Manuscript submitted January 3, 2011; revised manuscript received March 8, 2011. Published April 12, 2011.

It is well known that in semiconductor materials and device fabrication, the important issues are materials' purity, growth, defect control, lithography, etching, contact formation, and device performance. ${ }^{1,2}$ Among these, etching is an important process in which the grown material is required to be partially removed using specific patterns for subsequent doping, metalization, device structure formation, etc. In such processes, the rate of material removal has to be fast and repeatable. There is another associated process, in which the top layer of a material is cleaned before subsequent growth by removing a few angstroms and associated top layer contaminants. In this cleaning process, it is essential that the top layers are removed with care and the least amount of material is wasted. Thus, this chemical cleaning process has to be one with a slow material removal rate and with the least amount of interfering residues.

Such etching and cleaning may be done by electrochemical, wet chemical methods, reactive ion or sputter ion cleaning. ${ }^{3}$ Electrochemical processes are not always very selective while removing materials and often require very tight control over bias voltages and currents. Wet chemical etching is a method of removing the top layer of a material in a solvent leading to its dissolution in the reaction medium. Often the etchants are a combination of acids or a combination of bases. In wet chemical methods, with proper calibration, it is possible to retain better material crystalline quality of the etched material and by controlling the concentration of chemicals in the reaction, the material removal rate can also be rigorously controlled. However in all etch steps, residues from etching steps are present; these need to be understood to evaluate the likely effects on the subsequent electrical properties of the device created. Wet chemical etching is thus often the preferred etching mechanism for lithographic process. It has often been seen that the traditional wet chemical or electrochemical processes may also have their other limitations, even though they are quite cost effective. Inability to get high etching rates or reaction induced surface passivation are some of the associated problems. Reactive ion etching and sputter ion cleaning, also called dry etching, was developed to overcome such problems. These processes have relatively higher etch/erosion rates, depending on the material being removed and the reactive or sputtering gas used. But they also have their own limitations in terms of higher defect and crystalline damage creation and residue implantation. Over the years, this dry etching procedure, has often become the standard operating procedure for certain specialized semiconductor industries. ${ }^{4-6}$ Interestingly, wet chemistry continues to play an important role with RCA cleaning, chemical-mechanical polishing and copper deposition.

$\mathrm{GaN}$ and and associated materials are quite hard and reports of their wet etching are few, ${ }^{7}$ with most reports preferring the dry

\footnotetext{
${ }^{\mathrm{c}}$ Permanent address: National Physical Laboratory, New Delhi, 110012, India

E-mail: nkarar78@gmail.com
}

method, even though it requires large capital investments. ${ }^{4}$ However, the reported sputtering rates for GaN and related materials are also quite low and thus sputter etching is also time consuming. Photo-chemical and light induced electro-chemical etching techniques are other etching alternatives currently available for InGaN/ GaN related materials. ${ }^{8}$ In this context, we discuss the possibilities of etching InGaN/GaN type materials using wet chemical methods. The choice of acidic and basic mixtures to be used is an issue when an unknown material is to be wet chemically etched. There were literature reports on the use of etchants like (i) mixture of hot conc. $\mathrm{H}_{2} \mathrm{SO}_{4}$ and $\mathrm{H}_{3} \mathrm{PO}_{4}$ at $250^{\circ} \mathrm{C}$ (Ref. 9); (ii) $\mathrm{HCl}$; (iii) $\mathrm{NH}_{4} \mathrm{OH}$ (Ref. 10); but the usage and performance analysis of these last two in succession to combine the oxidizing and then the reducing features together was not envisaged earlier; that has been done here; (iv) usage of $\mathrm{HF}$ and $\mathrm{HNO}_{3}$ mixture based (isotropic and anisotropic) etchants was based on one of the authors' past experience of working on GaSb based materials. ${ }^{11}$ The etching performance of these chemical mixtures were then compared. X-ray photo electron spectroscopy (XPS) of the etched samples were performed to know the chemical contaminants present after the etch so that future results of electrical measurements can be appropriately correlated. This also gave information on the chemical bonding environments for $\mathrm{Ga}, \mathrm{In}, \mathrm{N}$, and $\mathrm{O}$ on the surface. Such an analysis, using the similarities of an earlier report ${ }^{12}$ will also help assign the XPS spectra.

\section{Experimental}

GaN and InGaN samples were prepared by organo-metallic chemical vapor deposition/epitaxy (MOCVD/MOVPE) process, with sapphire $\left(\mathrm{Al}_{2} \mathrm{O}_{3}\right)$ as a substrate material. The growth details are briefly the following: a base layer of undoped $\mathrm{GaN}$ was grown on the sapphire to a thickness so that the surface growth defect density stabilized and epitaxial layer growth was achieved. A semi-insulating $\mathrm{GaN}$ layer was grown on top of this layer to isolate future electronic structures. The total GaN layer thickness was at least 2000 $\mathrm{nm}$ or more. Often the GaN thickness was double of this. This buffer layer was required to get an appropriate lattice matching for the subsequent InGaN/GaN based active layers. Active semiconductor layers based on $\mathrm{p}$ and $\mathrm{n}$ type $\mathrm{InGaN}$ were grown on top of this (Fig. 1). ${ }^{13}$ The In content was about $17 \%$ or so. This active layer thickness varied from 150 to $450 \mathrm{~nm}$ or so, depending on the type of doping profile and structure needed.

As stated above, the objective here is to show the relative efficacy of different chemical etching processes on the top active InGaN layer (Fig. 1). The etchants studied are (i) anisotropic hydrofluoric acid (HF) having one part $\mathrm{HNO}_{3}, 69.8 \%$, one part $\mathrm{HF} 49 \%$ and three parts of de-ionized water $\left(\mathrm{H}_{2} \mathrm{O}\right)$, sourced from a Millipore water system), (ii) isotropic hydrofluoric acid (HF), (having nine parts of $\mathrm{HNO}_{3}, 69.8 \%$, and one part $\mathrm{HF} 49 \%$ ), (iii) first $\mathrm{NH}_{4} \mathrm{OH}$ 


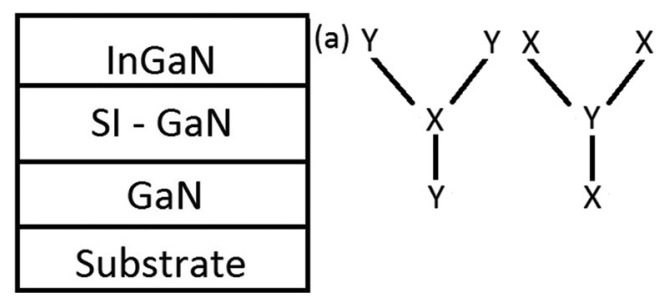

Figure 1. (a) A schematic diagram of the InGaN samples being etched; (b) A schematic diagram of the different bonds, associated with etched fcc InGaN structure; $\mathrm{X}$ represents $\mathrm{Ga}$ or $\mathrm{In}$; $\mathrm{Y}$ represents $\mathrm{N}$ or an etch related radical like $\mathrm{OEx}$ with $\mathrm{E}$ being an element like $\mathrm{H}, \mathrm{N}, \mathrm{P}, \mathrm{F}$ or $\mathrm{Cl}$ and $\mathrm{x}$ is a real positive fraction less than one.

soln. followed by concentrated hydrochloric acid (conc. $\mathrm{HCl}$ $37.5 \%)$, (iv) mixture of sulfuric acid $\left(\mathrm{H}_{2} \mathrm{SO}_{4}, 96.9 \%\right)$ and phosphoric acid $\left(\mathrm{H}_{3} \mathrm{PO}_{4}, 85 \%\right)(3: 1$ ratio), heated to a hot-plate temperature of $250^{\circ} \mathrm{C}$, and $(\mathrm{v})$ concentrated ammonia solution $\left(\mathrm{NH}_{4} \mathrm{OH}\right.$ soln. 29.5\%). All the chemicals were sourced from Fisher Scientific and used as is.

The etched surface morphology was observed using a Nikon Eclipse TE 2000-S optical microscope and the resulting images were analyzed using the open-source Image-J software. The sample surface was also observed using a Talystep surface profilometer as well as a Vecco Digital Instruments Nanoscope IV Multi-mode Atomic Force Microscope (AFM) in taping mode using a Nanoscope 6.12 Software. Fifty micrometre square of sample surface were considered for investigation in each case. However, such topographic images are omitted here for brevity and only the surface roughness values have been stated (Table I).

We tried out different polymers, including colorless nail polish, and Shiplay photoresists as mask materials. The samples were cured to increase the adhesion to the surface. In etching using HF, at the end of the etch process, the polymers had got totally removed, due to etching under the polymers and reaction with the polymers too. So properly calibrating the etch rate became an issue. The etch rate calibration was then done by partially dipping the samples into the etch solution so that a measurable step could be formed.

In that respect, mask materials like colorless nail polish, and Shiplay photoresists could significantly withstand the Ammonia solution/ $\mathrm{HCl}$ etch cycles for a long time and may be considered as an appropriate mask for this etch. However, we were unable to find an appropriate polymer based mask for the HF related wet etchants. It will be another totally different field of research to identify the appropriate polymer and its mean molecular weight/chain length from among polyethylene, difluoro-ethylene, and tryfluoro-ethylene, to see which one of these can withstand the etchant for the etching times required while slowly getting degraded in its own chain length and bond structure so that it becomes effectively removable at the end of the etch with the common solvents available in the laboratory.

Table I. Table showing the etch rates of different etchants on InGaN/GaN and their surface roughness values after etching; Unetched surfaces had RMS roughness values of about $27 \mathrm{~nm}$. This table is a partial one as the surface cleaning related etchants (ammonia and phosphoric acid) are evidently slow etchants, and their associated erosion rates were not calculated.

\begin{tabular}{lcc} 
Etchant & rate $(\mu \mathrm{m} / \mathrm{min})$ & RMS roughness $(\mathrm{nm})$ \\
\hline Anisotropic HF & 0.13 & 18.1 \\
Isotropic $\mathrm{HF}$ & 0.30 & 23.7 \\
Conc. $\mathrm{NH}_{4} \mathrm{OH}$ and conc. $\mathrm{HCl}$ & 0.04 (cycle) & 20.4 \\
Conc. $\mathrm{H}_{2} \mathrm{SO}_{4}$ and $\mathrm{H}_{3} \mathrm{PO}_{4}$ & low & 16.8 \\
$\mathrm{NH}_{4} \mathrm{OH}$ solution only & low & 4.7
\end{tabular}

In order to distinguish an etched depth with surface roughness, HF based etching was done for half an hour so that after washing the etched area was visible to the eye under a microscope. In the case of cyclic etching, each cycle consisted of dipping the sample first in ammonia solution, washing it and then dipping it in $\mathrm{HCl}$, each for 10 min duration. This cycle was repeated 10 times so that an appreciable amount of material was etched away and the etched thickness could be measured without any major uncertainty. In the case of cyclic etching, the associated number presented implies a minutes' cycle etch (Table I).

Surface chemical spectra was taken using a VG Escalab 220i-XL X-Ray Photoelectron Spectroscopy (XPS) system with a double crystal based monochromatic Al source using a pass energy of 20 $\mathrm{eV}$ for the high resolution scans with a step interval of $0.1 \mathrm{eV}$. The related data analysis and peak fitting was done using CasaXPS software ${ }^{14}$ and finally plotted using Origin software. The components of the raw elemental XPS scans were analyzed using a Shirley background, with all components having an equal full width at half maximum (FWHM) values and a profile $80 \%$ Gaussian and $20 \%$ Lorentzian curves. The number of assigned components if different than the number stated here lead to a difficulty in getting the obtained experimental profile. The XPS peak assignment was based on accepted standard literature reports. ${ }^{15-26} \mathrm{GaP}$ was used as a standard sample to understand the GaN XPS peaks better, since in Ga based compound semiconductor samples, very often there is interference of $\mathrm{Ga}$ related XPS and Auger peaks with other elemental peaks over a wide binding energy (BE) range.

\section{Results and Discussion}

Anisotropic HF etchant.-The details of different elemental peaks found after the etching processes, the different related bonds and associated peaks, their correlation and related analysis are presented below. Traces of organic contaminants in the form of carbon C 1 s peaks were visible at $284.6 \mathrm{eV}$ (Fig. 2a). This C-H bond based peak position was also used for a calibration of the other elemental peak positions.

The best possible peak fit for Ga 2p3/2 (Fig. 3a) suggests presence of three components at $1116.8,1118.1$ and $1119.7 \mathrm{eV}$ respectively, corresponding to $\mathrm{Ga}-\mathrm{N}$ bonds related peaks, the last peak is possibly Ga-oxyfluoride $\left(\mathrm{GaOF}_{x}\right)$ related, the latter coming from the etch process. ${ }^{23,24}$ The Ga-N related peak is relatively weaker due to the chemically etched surface. This fitted average Ga-N related peak and the normal peak for $\mathrm{Ga}-\mathrm{O}$ are a little off from their usual position, possibly due to the presence of the oxyfluoride component at the surface (Table II). Depending on the value of $x$, and the relative concetration of HF in the reactant, the relative position of the $\mathrm{GaOF}_{x}$ peak may shift a bit. As evident from above, Ga 2p3/2 has a high binding energy and thus correspond to low kinetic energy electrons. Thus, the above data came primarily from the upper part of the etched surface as opposed to the Ga $3 \mathrm{~d} 5 / 2$ peaks which have a very low binding energy and hence corresponds to electrons with higher kinetic energy.

Ga $3 \mathrm{~d} 5 / 2$ peak is in close proximity to In $3 \mathrm{~d} 5 / 2$ peaks. $^{20,25}$ The best fitted peak components of the overall profile were separated based on reported results for $\mathrm{Ga} 3 \mathrm{~d} 5 / 2$ and experimental data obtained after a comparison with data from a standard $\mathrm{GaP}$ sample (not shown here). The Ga $3 \mathrm{~d} 5 / 2$ had two peak components at 18.7 and $19.8 \mathrm{eV}$ corresponding primarily to Ga-N and the $\mathrm{Ga}^{-} \mathrm{ON}_{x}$ or Ga-O related component. It is difficult to separate the latter two here. The latter component is unlikely to be from $\mathrm{Ga}^{-} \mathrm{OF}_{x}$. The In $4 \mathrm{~d} 5 / 2$ associated peak at $16.5 \mathrm{eV}$ is attributed to In-N (Fig. 4a). The In $4 \mathrm{~d} 5 / 2$ could not be separated into components. These peaks correspond to deeper sample depths.

The observed change in the relative intensity of $\mathrm{Ga}-\mathrm{N}, \mathrm{Ga}-\mathrm{O}$ or for $\mathrm{Ga}-\mathrm{OF}_{x}$ related peak components with respect to other components for the "surface" and "bulk" part of the sample, i.e. a comparison of relative intensities of the Ga 2p3/2 and Ga $3 \mathrm{~d} 5 / 2$ components shows that etching processes are only surface sensitive. Thus based 

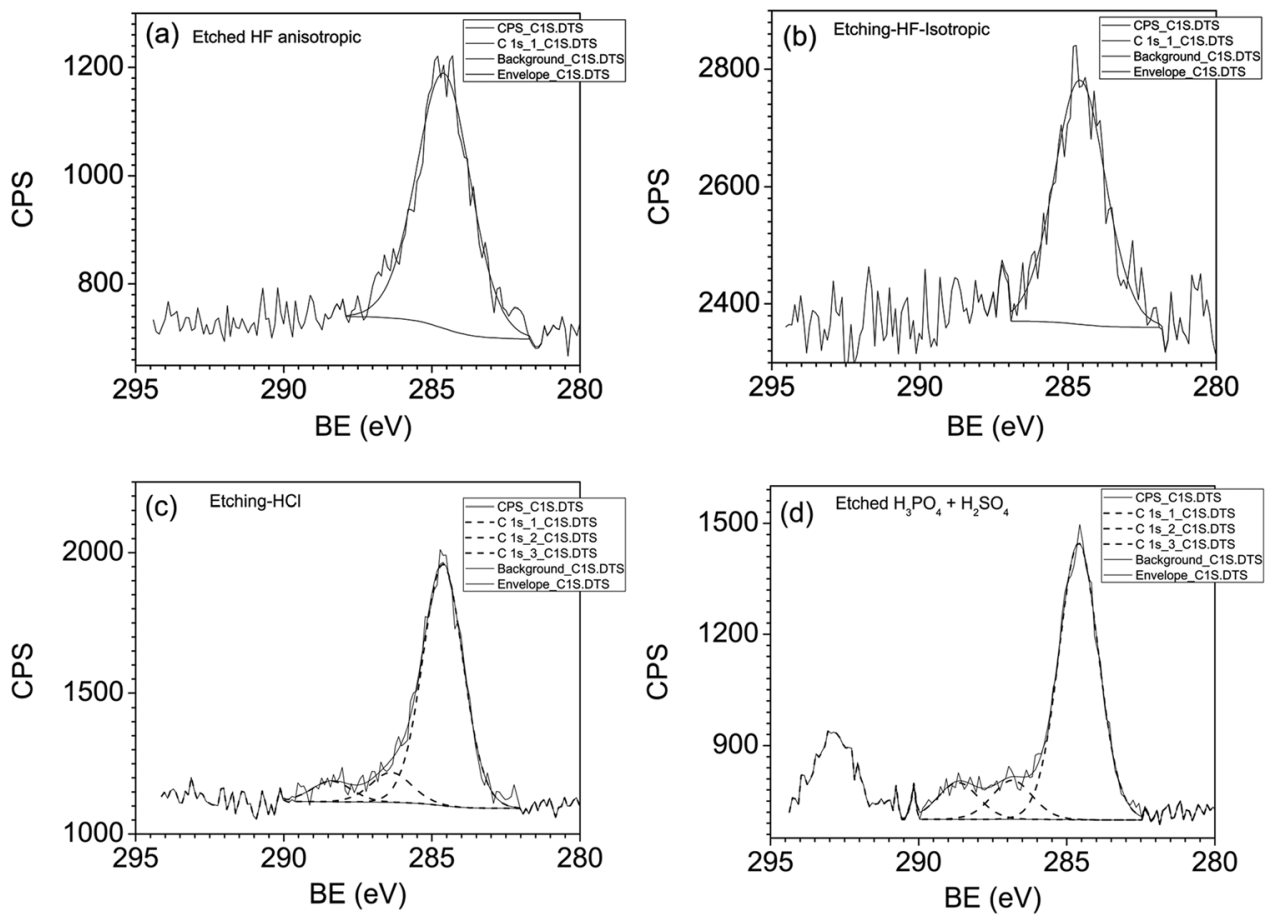

Figure 2. C 1s peak profile of different etched InGaN samples.

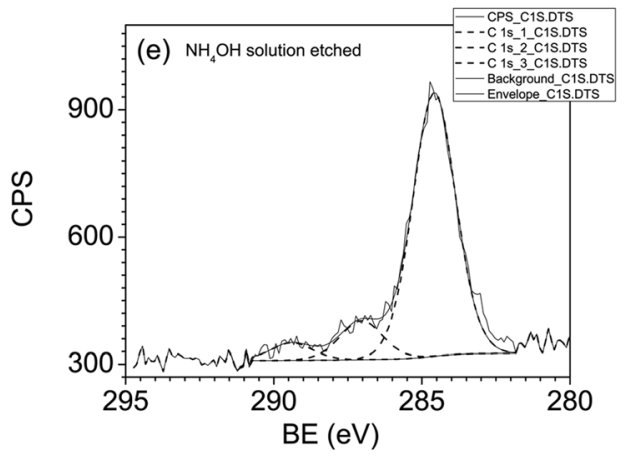

on above data, it may be said that the number of expected Ga Auger peaks can be taken as two or at most three. Any other fitted component in that contour may be safely considered to be from other elements.

In $3 \mathrm{~d} 5 / 2$ had two major peak components at 443.8 and $444.9 \mathrm{eV}$ corresponding to In-N bonds and In-O or In related oxyfluoride (In$\mathrm{OF}_{x}$ ) components, respectively (Fig. 5a). Since In 3d5/2 has high kinetic energy, the spectrum will be dominated by the bulk. These fitted peaks were also a little shifted from their expected positions due to the formation of the oxyfluoride component at the surface and our inability to separate the two (Table II).

A broad peak is observed at around $398 \mathrm{eV}$. This peak has two major constituents, one for the Ga LMM Auger lines and the other is for $\mathrm{N}$ 1s photoelectrons (Fig. 6a). ${ }^{21,22}$ There were two resolved peak components from $\mathrm{Ga}$ Auger lines corresponding to electrons with moderate kinetic energies - these were distinguished after comparison with data from a standard $\mathrm{GaP}$ sample. This standard GaP sample had no peaks for $\mathrm{N}$ 1s but two Ga LMM Auger lines for $\mathrm{Ga}-\mathrm{N}$ and surface oxidation related Ga-O, respectively. These Auger components were from surface of the material and correspond to the associated chemical states. Thus these have a correlation to the different $\mathrm{Ga} 2 \mathrm{p} 3 / 2$ or $\mathrm{Ga} 3 \mathrm{~d} 5 / 2$ peak components observed in the sample. These two components are quite broad and correspond to Ga-N and $\mathrm{Ga}-\mathrm{O} / \mathrm{GaOF}_{x}$; it was difficult to fit a third component here due to the profile contour. Based on the type of etching process used, the number of $\mathrm{Ga}$ Auger peak components varied in each case. The number of Ga Auger peak components also correlate to the number of Ga 2p3/2 and Ga 3d5/2 peak components. The N 1s peak components were best fit at 397.1 and $398.9 \mathrm{eV}$. The other remaining components were appropriate Ga Auger lines. The peak at $397.1 \mathrm{eV}$ is from $\mathrm{N}-\mathrm{Ga} / \mathrm{N}-\mathrm{In}$ bonds. A comparison to an earlier report on the analysis of $\mathrm{N} 1 \mathrm{~s}$ core level shifts in silicon oxynitride $\left(\mathrm{SiON}_{x}\right)$ (Ref. 12) shows that a possibility of $\mathrm{GaON}_{x} / \mathrm{Ga}-\left(\mathrm{NO}_{3}\right)_{3}$ formation from the etch process is quite likely here too. In that case, the higher binding energy component, i.e. the smaller peak at $398.9 \mathrm{eV}$ may correspond to the oxynitride component. The known electronegativity values for $\mathrm{Ga}$ is 1.6 , for $\mathrm{In}$ it is 1.7 , for $\mathrm{N}$ it is 3 and for $\mathrm{O}$ it is 3.5 (Refs. 27 and 28); thus it is most likely that due to the low difference in the electronegativity values for $\mathrm{Ga}$ and In, separate peaks for $\mathrm{Ga}$ and In are unlikely; but there may be a peak corresponding to $\mathrm{O}$, due to its distinctly greater electronegativity.

The oxygen $\mathrm{O} 1 \mathrm{~s}$ peak for this sample could not be resolved into components corresponding to difference between oxides and hydroxides of the metal $\mathrm{Ga}$ or In or for any oxyfluoride component as well, in part due to the weak signal of the O 1s peak (Fig. 7a). ${ }^{26}$ Traces of the HF etchant were visible as an XPS peak of $F$ 1s at $685.1 \mathrm{eV}{ }^{18,19}$ But it is not possible to further resolve this peak from $\mathrm{Ga}-\mathrm{F} / \mathrm{In}-\mathrm{F}$ or any oxyfluoride component due to its low intensity. 

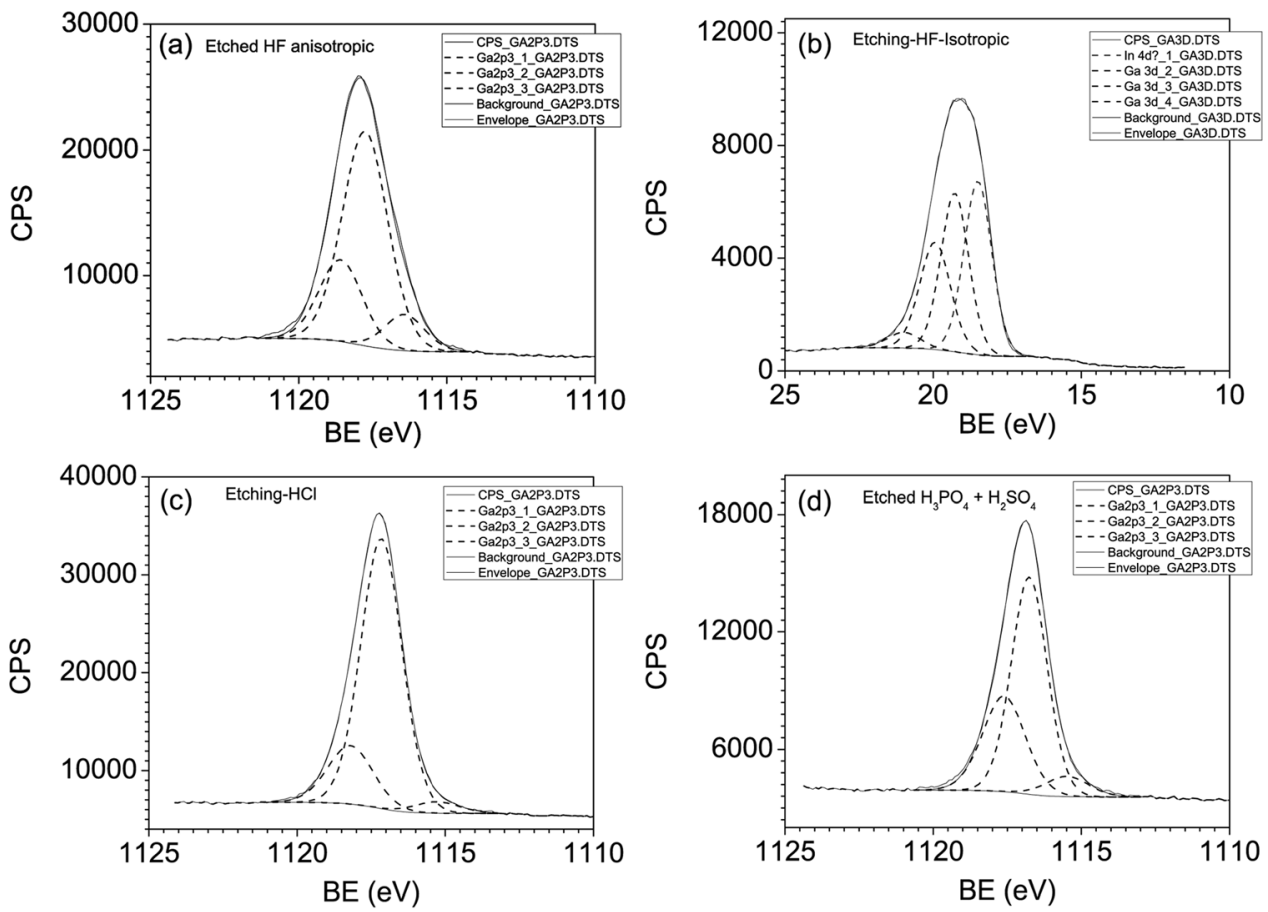

Figure 3. Ga 2 p3/2 peak profile of different etched InGaN samples.

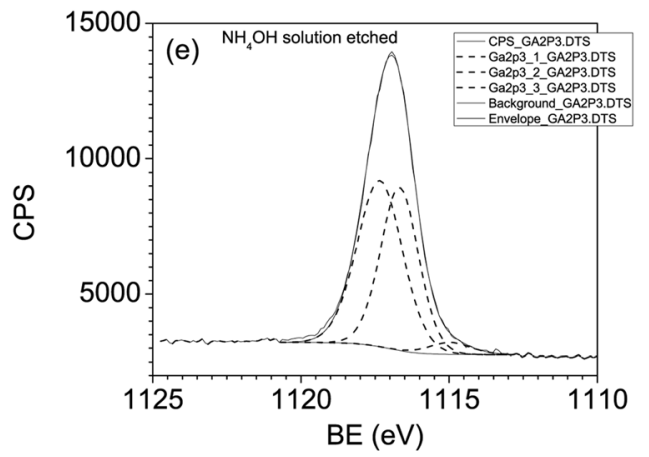

This HF based etching of InGaN sample also revealed two unknown low intensity peaks at 566.4 and $574 . \mathrm{eV}$, which may correspond to incorporation of chromium $(\mathrm{Cr} 2 \mathrm{p})$ from the $\mathrm{Al}_{2} \mathrm{O}_{3}$ sapphire substrate by diffusion during the high temperature growth process (peaks not shown here for brevity). Based on the above observation, where chromium diffusing was observed after etching of the original material, this etch was thought to be a moderately fast wet etch process. Its quantification may be beneficial for any future etching related process applications. The calibrated etch rates for this etchant at room temperature was $0.13 \mu \mathrm{m} / \mathrm{min}$

Isotropic HF etchant.- A single carbon C 1s peak at $284.6 \mathrm{eV}$ suggested presence of trace ambient contaminants (Fig. 2b).

The $\mathrm{Ga} 2 \mathrm{p} 3 / 2$ peak profile shows four related components at $1115.3,1116.7,1117.6,1119 \mathrm{eV}$ corresponding to $\mathrm{Ga}-\mathrm{N}, \mathrm{Ga}-\mathrm{O}$, $\mathrm{Ga}-\left(\mathrm{NO}_{3}\right)_{3} / \mathrm{GaON}_{x}$ and $\mathrm{Ga}-\mathrm{F} / \mathrm{oxy}$ fluoride $\left(\mathrm{Ga}-\mathrm{OF}_{x}\right)$ respectively

Table II. A table based on the above analysis and discussion showing the different XPS peak positions (within $\pm 0.2 \mathrm{eV}$ ) of different ion components; $\mathrm{E}$ refers to any non-halogen element, e.g., $\mathrm{P}, \mathrm{H}, \mathrm{N}$ etc. and $\mathrm{x}$ is a fraction; if $\mathrm{E}$ is a halogen, the related binding energy values are higher; the $\mathrm{O}$-and $\mathrm{OE}_{x}$ related peaks are not always distinguishable separately in each sample.

Components Peak positions $(\mathrm{eV})$

$\begin{array}{cc}\mathrm{Ga}-\mathrm{N} & 1115.1 \\ \mathrm{Ga}-\mathrm{O} & 1116.8 \\ \mathrm{Ga}^{\mathrm{O}} & 1117.8\end{array}$

In $3 \mathrm{~d} 5 / 2$

$\begin{array}{cc}\text { In-N } & 441 \\ \text { In-O } & 443.8 \\ \text { In-OE }_{x} & 444.9\end{array}$

$\mathrm{N} 1 \mathrm{~s}$
Ga-N/In-N Ga-ON $/$ /In-ON ${ }_{x}$
Ga 3d5/2

Components

Ga-N

Ga-O

$\mathrm{Ga}-\mathrm{OE}_{x}$

In $4 d 5 / 2$

In-N

In-O

O 1s

397

398.7
3.8

4.9$$
\mathrm{In}-\mathrm{OE}_{x}
$$$$
\text { Ga-O/In-O }
$$$$
\mathrm{Ga}-\mathrm{OE}_{x}
$$

\section{6}

15.2

17.1

17.4

530.8

532.2 

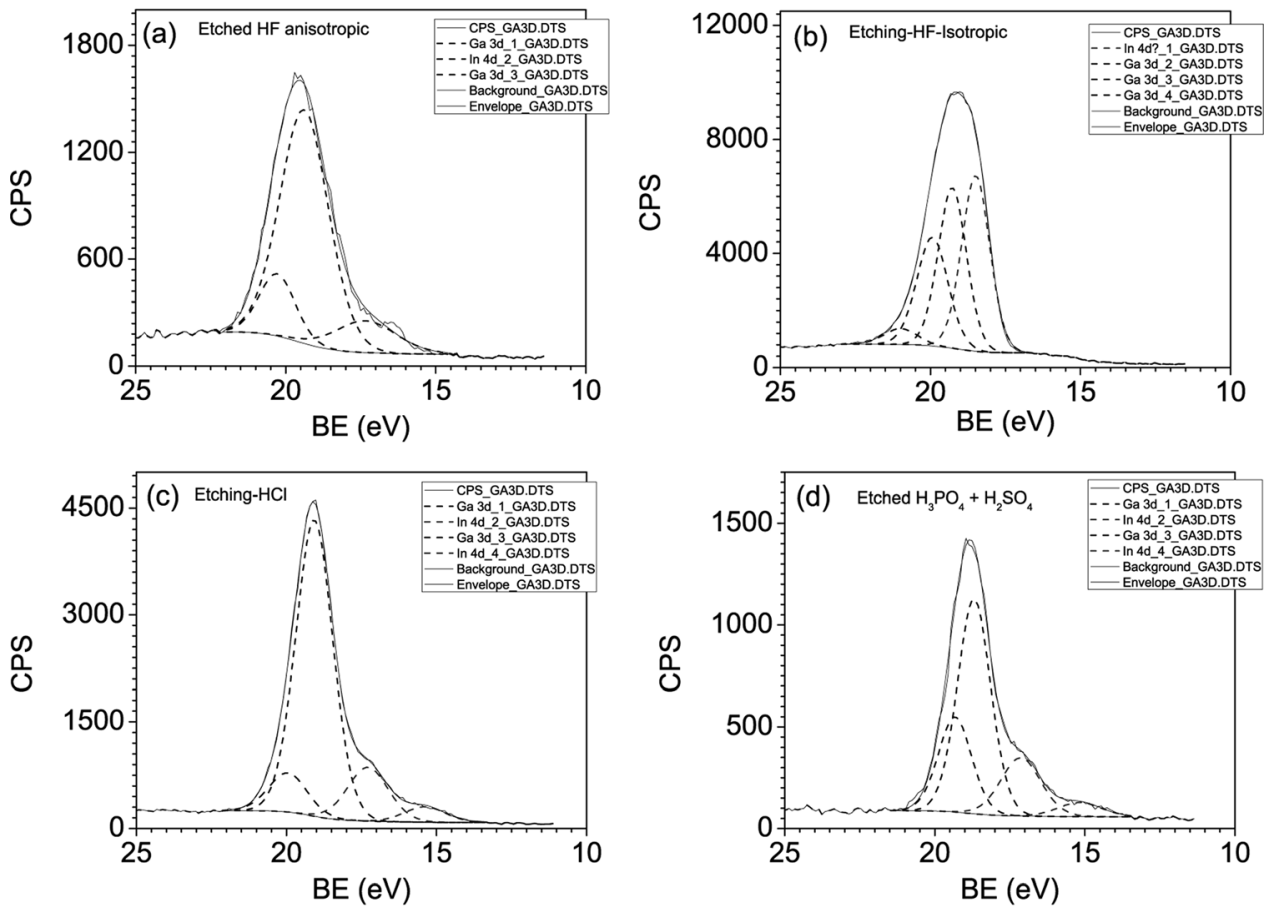

Figure 4. Ga 3d/In4d peak profile of different etched InGaN samples.

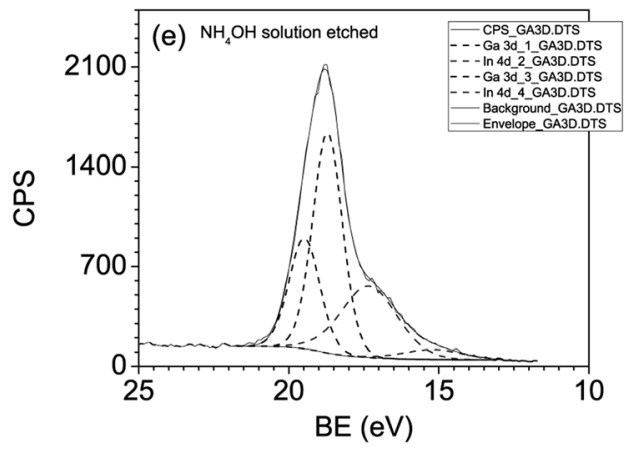

(Fig. 3b). The effect of change in the relative fluoride ion ratio in the etch solution on the peak position of $\mathrm{Ga}-\mathrm{OF}_{x}$ is notable.

The uniqueness of this etch was that four Ga $3 \mathrm{~d} 5 / 2$ peak components were visible at $18.4,19.1,19.8$, and $20.9 \mathrm{eV}$ possibly corresponding to $\mathrm{Ga}-\mathrm{N}, \mathrm{Ga}-\mathrm{O}, \mathrm{Ga}-\left(\mathrm{NO}_{3}\right)_{3} / \mathrm{GaONx}$ and $\mathrm{Ga}-\mathrm{F} /$ oxyfluoride $\left(\mathrm{Ga}-\mathrm{OF}_{x}\right)$ respectively (Fig. $\left.4 \mathrm{~b}\right)$. There are also two oxygen peaks to suggest possible presence of $\mathrm{NO}_{3}$. The $\mathrm{Ga}-\left(\mathrm{NO}_{3}\right)_{3} / \mathrm{GaON}_{x}$ peaks were visible separately due to the higher $\mathrm{NO}_{3}$ ion content in the etch solution. A comparison of the relative peak intensity changes for the different peak components related to Ga 2p3/2 and Ga 3d5/2 shows that the top layer contains relatively more intense peaks for the oxides while at the more deeper parts of the sample, the nitride peak component is predominant.

This InGaN sample does not show any presence of In $4 \mathrm{~d} 5 / 2$ peaks, or presence of any In $3 \mathrm{~d} 5 / 2$ peaks either. This suggests that all the InGaN layers was etched away leaving only the bottom GaN seed layers instead.

There were two N 1s peaks at $397 \mathrm{eV}$ and $398.5 \mathrm{eV}$ corresponding to $\mathrm{Ga}-\mathrm{N}$ and $\mathrm{Ga}-\left(\mathrm{NO}_{3}\right)_{3} / \mathrm{GaON}_{x}$ bonds respectively (Fig. 6b). The other peaks correspond to four Auger lines for the Ga peaks discussed below. The oxygen $\mathrm{O} 1 \mathrm{~s}$ peak for this HF etched sample had two components at 530.8 and $532.3 \mathrm{eV}$ possibly corresponding to $\mathrm{Ga}-\mathrm{O}$ and $\mathrm{Ga}-\mathrm{NO}_{3} / \mathrm{GaON}_{x}$ respectively (Fig. 7b). ${ }^{26}$ The similar In related components could be merged into the Ga related oxide spectra. Traces of the HF etchant in terms of an XPS peak of $F$ 1s at $684.9 \mathrm{eV}$ were also visible.
This HF etched InGaN sample also showed two low concentration peaks at 563.8 and $572.3 \mathrm{eV}$, which may correspond to a incorporation of chromium from the sapphire substrate.

In this sample, the upper InGaN layer was etched away due to the higher $\mathrm{NO}_{3}$ ion content in the etchant. The role of $\mathrm{HF}$ is to convert the top layer to insoluble $\mathrm{GaF}_{3} / \mathrm{InF}_{3}$, while $\mathrm{HNO}_{3}$ converts the fluoride to soluble gallium nitrate $\left(\mathrm{Ga}\left(\mathrm{NO}_{3}\right)_{3}\right)$ /indium nitrate $\left(\mathrm{In}\left(\mathrm{NO}_{3}\right)_{3}\right)$. Essentially, the amount of the fluoride in the medium remains constant, with $\mathrm{HF}$ acting more like a catalyst. The relative amount of $\mathrm{NO}_{3}$ ions in the solution determines the extent of etching. The calibrated etch rates for this etchant at room temperature was $0.30 \mu \mathrm{m} / \mathrm{min}$.

Conc. $\mathrm{NH}_{4} \mathrm{OH}$ and conc. $\mathrm{HCl}$ in succession.-This etch process was developed after observing the report of three independent groups ${ }^{10,16,17}$ and thereafter appropriately modifying the process. Traces of extra carbon peaks, other than the $\mathrm{C} 1 \mathrm{~s}$ at $284.6 \mathrm{eV}$ were observed, and corresponds to presence of adventitious contaminants (Fig. 2c).

The sample had three $\mathrm{Ga} 2 \mathrm{p} 3 / 2$ peak components at 1115.4 , 1117.1 and $1117.8 \mathrm{eV}$ corresponding to Ga-N bonds, Ga-O and possibly from $\mathrm{GaOCl}_{x}$ respectively (Fig. 3c). The peak for $\mathrm{Ga}-\mathrm{O}$ was strongest due to the nature of the etch process and the surface sensitive nature of this etch.

Ga $3 \mathrm{~d} 5 / 2$ region of this sample, which corresponds to signals from the deepest part within the material had peaks at 19.1 and 20 

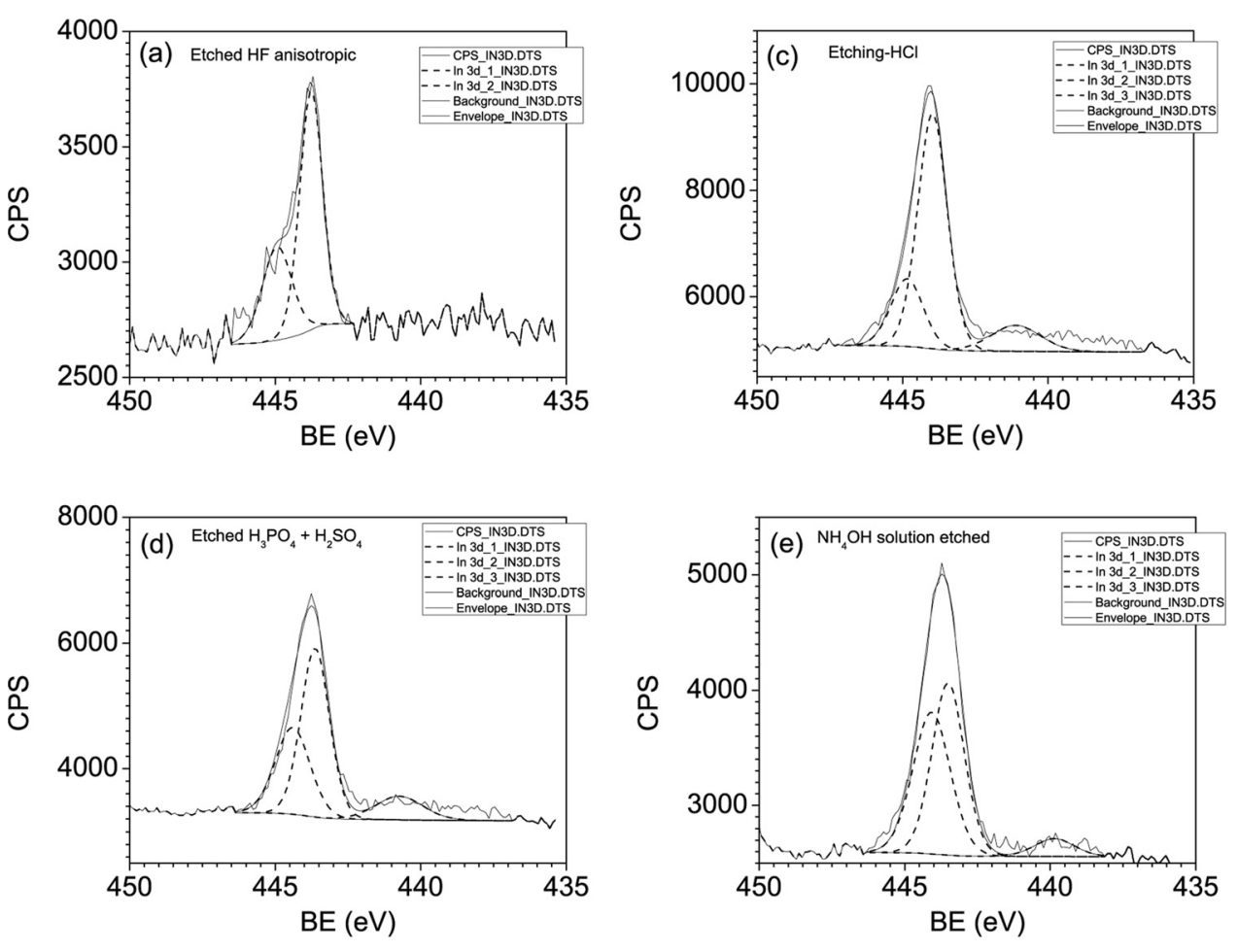

Figure 5. In $3 d 5 / 2$ peak profile of different etched InGaN samples; date from (b) was omitted due to complete In removal.
$\mathrm{eV}$ due to $\mathrm{Ga}-\mathrm{N}$ bonds and $\mathrm{Ga}-\mathrm{O}$ respectively (Fig. 4c). Looking at the center of the Ga-O peak here (Table II), it is felt that there may also be an unresolved $\mathrm{Ga}-\mathrm{OCl}_{x}$ component within it. This again suggests on the sensitive nature of the etch process. In $4 \mathrm{~d} 5 / 2$ showed two peaks at 15.2 and and $17.4 \mathrm{eV}$ corresponding to In-N bonds and In-O. The relative difference in the heights may correspond to the relative differences of reactivity of In and $\mathrm{Ga}$ with oxygen.

In $3 \mathrm{~d} 5 / 2$ has three components at $442,444.1$, and $445.4 \mathrm{eV}$ corresponding to In-N bonds, In-O and possibly $\mathrm{InOCl}_{x}$ respectively (Fig. 5c). The difference in the number of surface related In $3 d 5 / 2$ and volume related In $4 \mathrm{~d} 5 / 2$ peak components is due to the surface sensitive nature of the etch process. Nitrogen has two $\mathrm{N}$ 1s peaks at 396.8 and $397.6 \mathrm{eV}$ corresponding to $\mathrm{Ga}-\mathrm{N} / \mathrm{In}-\mathrm{N}$ bonds and $\mathrm{H} / \mathrm{OH}$ type bond formation respectively. There were also three Ga Auger peaks in the same area corresponding to Ga-N, Ga-O and GaOClx respectively (Fig. 6c). Oxygen $1 \mathrm{~s}$ showed peaks at $530.8 \mathrm{eV}$ from $\mathrm{Ga}-\mathrm{O} / \mathrm{In}-\mathrm{O}$ and at $531.9 \mathrm{eV}$ from $\mathrm{GaOCl}_{x}$ (Fig. 7c). No peak component for $\mathrm{Ga}-\mathrm{OH}$ could be separately identified, possibly due to the subsequent $\mathrm{HCl}$ related reaction. $\mathrm{Cl} 1 \mathrm{~s}$ peaks are not shown here for brevity.

In this two step etch process, at first gallium oxide $\left(\mathrm{Ga}_{2} \mathrm{O}_{3}\right)$ was formed using concentrated ammonia solution. This is in itself a limiting process as gallium oxide formation passivated the surface and stops further reactions. However, not much material is removed in this step as the oxide is in itself not very soluble. Thereafter, conc. $\mathrm{HCl}$ efficiently converted the gallium oxide to soluble gallium chloride, which was dissolved in the reaction medium. The top surface was then again oxidized for subsequent removal as a chloride and such number of cycles could be repeated as per requirement. This sample showed deep etching due to two the successive etching steps. A few $\mu \mathrm{m}$ could be etched by this process if used cyclically, with an etch rate of $0.04 \mu \mathrm{m} / \mathrm{min}$. It is thus seen that wet chemical etching can also be a stand alone process for material removal of InGaN type materials. The uniqueness of this process was the two steps required, at first the oxide formation with a solution and then its removal. It is felt that such two step process of etching may be subsequently emulated for other materials as well, specially where a single step etching gets limited by a surface passivation layer.
After etching, two peaks corresponding to $\mathrm{Cr} 2 \mathrm{p}$ were also visible at around 574 and $565.3 \mathrm{eV}$ in the form of two large humps due to the deep etching possible. These are possibly from $\mathrm{Cr}$ based impurities that out-diffuse from the sapphire substrate due to the sample growth temperature (spectra not shown here).

Conc. $\mathrm{H}_{2} \mathrm{SO}_{4}$ and $\mathrm{H}_{3} \mathrm{PO}_{4}$ at $250^{\circ} \mathrm{C}$.- This process was essentially based on the report by Weyher et al. ${ }^{9}$ and was used here for a comparison of the relative efficacy of different etchants. There were small adventitious trace contaminants other than the $\mathrm{C}-\mathrm{H}$ peak at $284.6 \mathrm{eV}$ along with a small peak for potassium $\mathrm{K} 2 \mathrm{p} 3 / 2$-which possibly came from the glassware at the elevated etching temperature of $250^{\circ} \mathrm{C}$ (Fig. 2d).

There were three $\mathrm{Ga} 2 \mathrm{p} 3 / 2$ peak components at 1115.1, 1116.8 and $1118 \mathrm{eV}$ corresponding to Ga-N bonds, Ga-O, and $\mathrm{Ga}-\left(\mathrm{PO}_{3}\right) /$ $\mathrm{Ga}^{-} \mathrm{OP}_{x}$ respectively (Fig. $3 \mathrm{~d}$ ). The $\mathrm{Ga}-\mathrm{O}$ peak was relatively strong corresponding to the nature of the etch process.

Peak components of $\mathrm{Ga} 3 \mathrm{~d} 5 / 2$ for $\mathrm{Ga}-\mathrm{N}$ bonds at $18.7 \mathrm{eV}$ and $\mathrm{Ga}-\mathrm{O} / \mathrm{Ga}-\mathrm{OP}_{x}$ at $19.3 \mathrm{eV}$ were also visible. The slight change from the peak position for any likely Ga $3 \mathrm{~d} 5 / 2$ for Ga-O may be noted (Table II). There were also two adjacent In $4 \mathrm{~d} 5 / 2$ peak components at 15.1 and $17.1 \mathrm{eV}$ corresponding to In-N bonds and In- $\left(\mathrm{PO}_{3}\right) / \mathrm{In}-\mathrm{O}$ respectively (Fig. 4d). The nature of this process ensured presence of relatively higher oxygen components on the surface.

In $3 \mathrm{~d} 5 / 2$ has three perceptible components at $441,443.8,444.9 \mathrm{eV}$ corresponding to In-N bonds, In-O and In-( $\left.\mathrm{PO}_{3}\right) / \mathrm{In}-\mathrm{OPx}$ (Fig. $5 \mathrm{~d}$ ) respectively.

Nitrogen N 1s had peak components at 396.4 for Ga-N/In-N bonds and at $397.7 \mathrm{eV}$ possibly for $\mathrm{ON}_{x}$ bonds. The lower values here for the $\mathrm{N} 1 \mathrm{~s}$ binding energy in $\mathrm{Ga}-\mathrm{N} / \mathrm{In}-\mathrm{N}$ is possibly due to lack of any other ion in the bond. In the same region, there were three $\mathrm{Ga}$ Auger peaks corresponding to $\mathrm{Ga}-\left(\mathrm{PO}_{3}\right), \mathrm{Ga}-\mathrm{O}$ and $\mathrm{Ga}-\mathrm{N}$ respectively (Fig. 6d).

There was also the two $\mathrm{O} 1 \mathrm{~s}$ components for $\mathrm{Ga}-\mathrm{O}$ and $\mathrm{Ga}-$ $\left(\mathrm{PO}_{3}\right) / \mathrm{In}-\left(\mathrm{PO}_{3}\right)$ at 531.3 and $532.5 \mathrm{eV}$ (Fig. 7d). There were traces of phosphorus $\mathrm{P} 2 \mathrm{p} 3 / 2$ at $133.3 \mathrm{eV}$ possibly from the above etchant residues. The role of heated $\mathrm{H}_{3} \mathrm{PO}_{4}$ was to convert the surface to $\mathrm{Ga}\left(\mathrm{PO}_{3}\right)_{3}$ while the $\mathrm{H}_{2} \mathrm{SO}_{4}$ converted this phosphate to soluble 

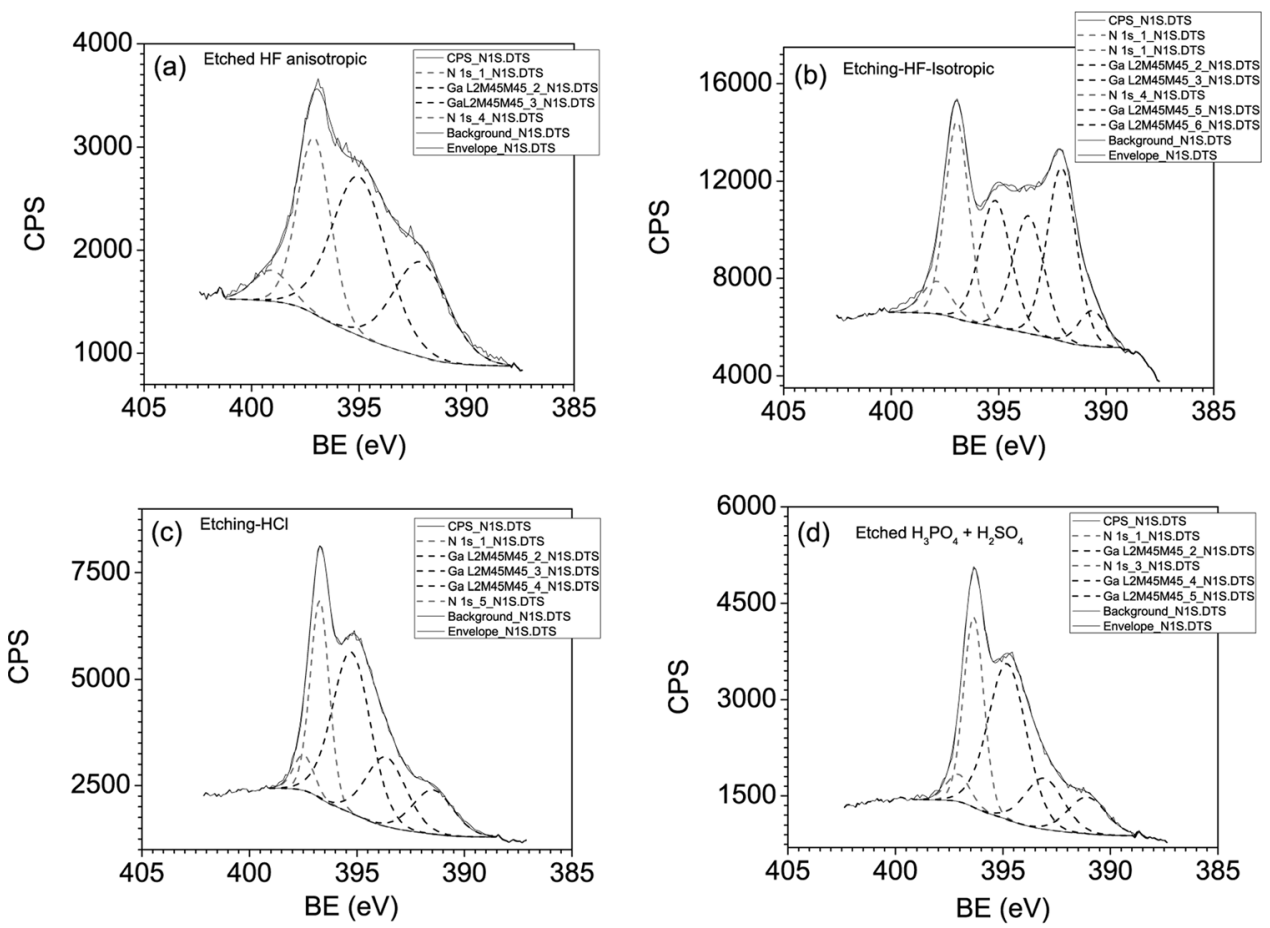

Figure 6. $\mathrm{N}$ 1s peak profile of different etched InGaN samples and from a standard GaP sample (f).
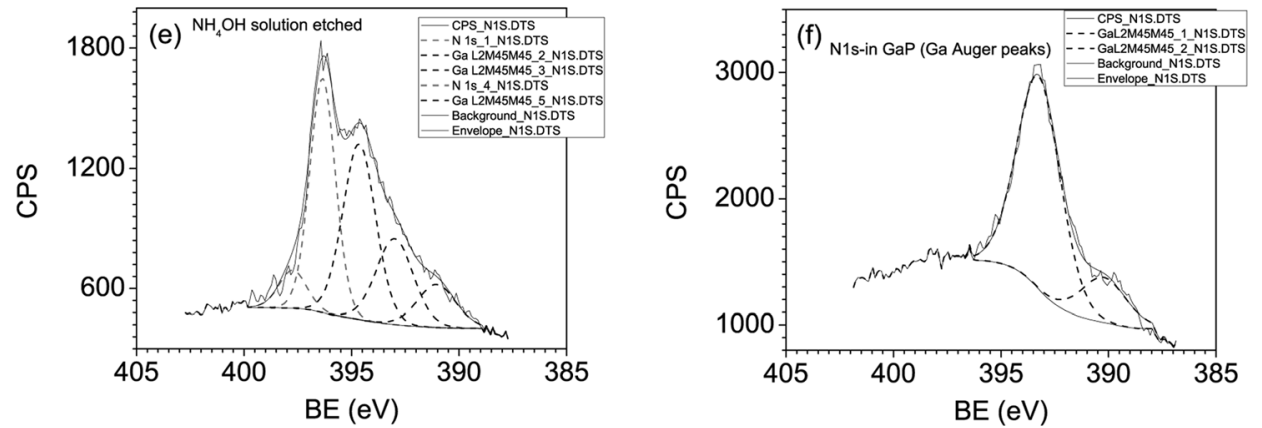

gallium sulfate. Here the etch rates were not calculated as this slow limiting process is essentially suitable only for surface cleaning.

$\mathrm{NH}_{4} \mathrm{OH}$ solution only. - This etch solution was separately considered in order to understand its performance as a stand alone etchant. There were traces of adventitious contaminants other than the $\mathrm{C}$ 1s C-H peak at $284.6 \mathrm{eV}$ (Fig. 2e).

The gallium $\mathrm{Ga} 2 \mathrm{p} 3 / 2$ had three resolvable components at $1114.9,1116.9$ and $1117.9 \mathrm{eV}$ corresponding to Ga-N bonds, Ga-O, and possibly $\mathrm{Ga}-\mathrm{ON}_{x}$; these signals came mainly from the surface (Fig. 3e). The oxygen component was strong due to the nature of the etchant. Two Ga $3 \mathrm{~d} 5 / 2$ peak components were visible at 18.7 and $19.5 \mathrm{eV}$ corresponding to $\mathrm{Ga}-\mathrm{N}$ bonds and $\mathrm{Ga}-\mathrm{O}$; the latter peak could not be further distinguished. There were also two related In $4 \mathrm{~d} 5 / 2$ peak components in the same area at 15.2 and $17.3 \mathrm{eV}$ related to In-N bonds and In-O respectively (Fig. $4 \mathrm{e}$ ).

There were three perceptible indium In $3 \mathrm{~d} 5 / 2$ peak components at $440,443.7$ and $445 \mathrm{eV}$ corresponding to In-N bonds, In-O and InONx respectively (Fig. 5e).

Nitrogen N 1s had peak components at $396.4,398.3 \mathrm{eV}$ corresponding to Ga-N/In-N bonds and $\mathrm{ON}_{x}$ bonds respectively; the slight shift of the peak due to higher presence of $\mathrm{ON}_{x}$ may be noted. There were also three Ga Auger peaks in the same area corresponding to $\mathrm{Ga}-\mathrm{N}, \mathrm{Ga}-\mathrm{O}$, and $\mathrm{Ga}-\mathrm{OH}$ respectively (Fig. 6e).

There were two perceptible oxygen $\mathrm{O} 1 \mathrm{~s}$ peak components at 530.4 and $532 \mathrm{eV}$ corresponding to $\mathrm{Ga}-\mathrm{O} / \mathrm{In}-\mathrm{O}$ and $\mathrm{GaON}_{x} / \mathrm{InON}_{x}$ respectively from the usage of oxidizing etch $\mathrm{NH} 4 \mathrm{OH}$ (Fig. 7e). The sole presence of ammonia solution and related reaction and identification of a separate $\mathrm{Ga}-\mathrm{ON}_{x}$ related peak resulted in a slightly different fitted $\mathrm{O}$ 1s peaks here (Table II). Gallium oxide $\left(\mathrm{Ga}_{2} \mathrm{O}_{3}\right)$ related bond formation led to passivation of the top surface and limited further material removal. Here etch rates were not calculated as this slow process is suitable only for surface cleaning.

Other etching issues.-A detailed surface analysis using optical as well as atomic force microscopy on the different etched surfaces was done. The etch pits were visible for all etchants and in spite of the large number of areas and images considered for each type of etching, it is difficult to say that only a particular etchant is useful in revealing the etch pits, though the nature of the etch pits were seen to depend on the type of etchant. This is unlike the situation of other known III-V materials, where certain etchants are known to reveal etch pits much better than others. InGaN/GaN has a much higher defect density wrt other III-V materials. The effect of an etchant on a particular material is also dependent on the type of etchant under consideration, on the crystal structure of the material and relative erosion rate of different crystalline planes of that material. So a plane selective etchant for a certain material may not be that plane sensitive in another.

Though wet etching by cyclic ammonia/ $\mathrm{HCl}$ is slower than the two HF based etchants studied, it is not so corrosive to remove the polymer masks as well. Often, etching is time barred, where after a 

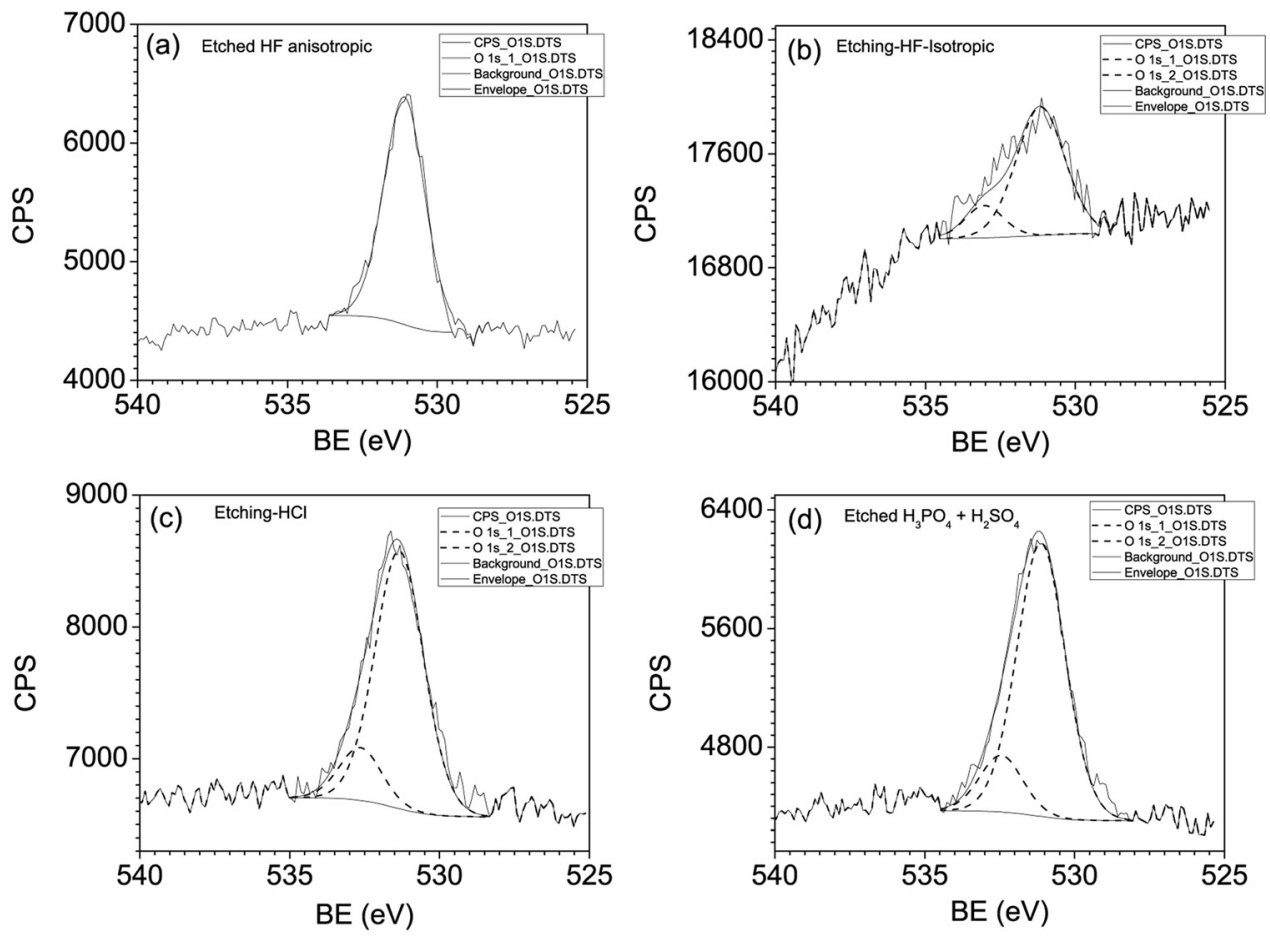

Figure 7. O 1s peak profile of different etched InGaN samples. The fluoro-oxide related peaks, if any, have not been accounted for.

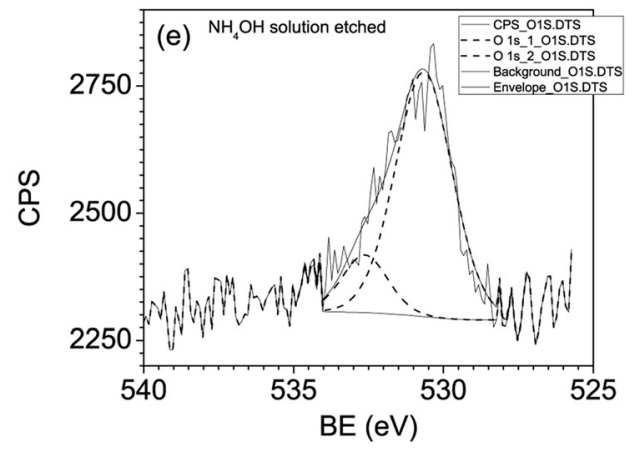

while the etch process stops on its own. In this two step process, the passivating oxide formed in the initial step is removed in the next step. This is such a cyclic two step process, where effectively a good amount of material may be removed. Such a two step process may perhaps be replicated for other materials as well. It may be recalled that due to the time barring nature of many wet etch processes, sputter-etching processes became popular. Such ideas may thus revive interest in wet etching processes again. Usage of this process in low volume applications may be possible.

\section{Conclusions}

XPS results for different etched InGaN/GaN surfaces showed the chemical state of different surfaces after etching, including the different impurities present. This could be related to the efficiency of the etching process. A comparative analysis of different studied etchants shows that cyclic usage of conc. ammonia $\left(\mathrm{NH}_{4} \mathrm{OH}\right)$ solution followed by conc. $\mathrm{HCl}$ is a "clean" etchant for removing InGaN/GaN layers. A similar two step etching process may be successful for other materials where etching is limited by surface passivation. $\mathrm{HF}$ and $\mathrm{HNO}_{3}$ solution mixture was also successful as a relatively fast etchant. It was often possible to etch almost up to the base $\mathrm{GaN}$ layer. Etchants like ammonia $\left(\mathrm{NH}_{4} \mathrm{OH}\right)$ solution alone or Conc. $\mathrm{H}_{2} \mathrm{SO}_{4}$ and $\mathrm{H}_{3} \mathrm{PO}_{4}$ mixture were good enough only for cleaning up the top layer. The etch rates were also calibrated. However, compared to other III-V materials, the etching rate in $\mathrm{GaN} / \mathrm{InGaN}$ is an order of magnitude less.

\section{Acknowledgments}

One of the authors' (NK) thank the Indo-US Science and Technology Forum for providing him a fellowship for this work. B. Jampana (UD) is thanked for allowing us to use his samples.

University of Delaware assisted in meeting the publication costs of this article.

\section{References}

1. Introduction to Nitride Semiconductor Blue Lasers and Light Emitting Diodes, S. Nakamura and S. Chichibu, Editors, CRC, USA (2000).

2. Group III Nitride Semiconductor Compounds, B. Gill, Editor, p. 35, Oxford University, New York (1998).

3. Handbook of Semiconductor Wafer Cleaning Technology, W. Kern, Editor, Noyes Publications, USA (1993).

4. Handbook of Plasma Processing Technology, S. Rossnagel, J. Cuamo, and W. Westwood, Editors, Noyes Publications, USA (1990).

5. Y. Kawakami, A. Kaneta, L. Su, Y. Zhu, K. Okamoto, M. Funato, A. Kikuchi, and K. Kishino, J. Appl. Phys., 107, 023522, (2010).

6. M. Schuette and W. Lu, J. Vac. Sci. Technol. B, 25, 1870, (2007).

7. D. Zhuang and J. H. Edgar, Mater. Sci. Eng. R, 48, 1, (2005).

8. A. Tamboli, M. Schmidt, S. Rajan, J. Speck, U. Mishra, S. DenBaars, and E. Hua, J. Electrochem. Soc., 156, H47, (2009).

9. J. L. Weyher, S. Lazar, L. Macht, Z. Liliental-Weber, R. Molnar, S. Muller, V. Sivel, G. Nowak, and I. Grzegory, J. Crystal Growth, 305, 384, (2007).

10. R. Sohal, P. Dudek, and O. Hilt, Appl. Surf. Sci., 256, 2210, (2010) 
11. N. Karar, S. Basu, and S. Sainkar, J. Alloys Compd., 307, 272, (2000).

12. J. Eng, I. Hubner, J. Barriocanal, R. Opila, and D. Doren, J. Appl. Phys., 95, 1963, (2004).

13. N. Faleev, B. Jampana, O. Jani, H. Yu, R. Opila, I. Ferguson, and C. Honsberg, Appl. Phys. Lett., 95, 051915, (2009).

14. The Casa Cookbook, N. Fairley and A. Carrick, Editors, Acolyte Science, UK (2005).

15. Handbook of X-Ray Photoelectron Spectroscopy, J. Moulder, W. Stickle, P. Sobol, and K. Bomden, Editors, Physical Electronics Inc., USA (1995).

16. Y. Chang, H. Chiu, Y. J. Lee, M. Huang, K. Lee, M. Hong, Y. Chiu, J. Kwo, and Y. Wang, Appl. Phys. Lett., 90, 232904, (2007).

17. H. Lu, X. A. Cao, S. F. LeBoeuf, H. C. Hong, E. B. Kaminsky, and S. D. Arthur, J. Crystal Growth, 291, 82, (2006).

18. A. Vajpai, S. Chua, S. Tripathi, and E. Fitzgerald, Appl. Phys. Lett., 91, 08311, (2007).

19. Y. Choi, J. Lim, Y. Kim, M. Kim, O. Seok, and M. Han, ECS Trans., 25(12), 117 (2009).
20. M. Toda, J. Yanagisawa, K. Gamo, and Y. Akasaka, J. Vac. Sci. Technol. B, 22, 3012, (2004).

21. H. Hasegawa, T. Inagaki, S. Ootomo, and T. Hashizume, J. Vac. Sci. Technol. B, $21,1844,(2003)$.

22. T. Nagata, G. Koblmller, O. Bierwagen, C. Gallinat, and J. Speck, Appl. Phys. Lett., 95, 132104, (2009).

23. S. Wolter, B. Luther, D. Waltemyer, C. Onneby, S. Mohneya, and R. Molnar, J. Appl. Phys., 70, 2156, (1997).

24. M. Toda, J. Yanagisawa, K. Gamo, and Y. Akasaka, J. Vac. Sci. Technol. B, 22, 3012, (2004).

25. A. Siderenko, H. Peisert, H. Neumann, and T. Chasse, Surf. Sci., 601, 4521, (2007).

26. T. Hashizume, S. Ootomo, R. Nakasaki, and S. Oyama, and M. Kihara, Appl. Phys. Lett., 76, 2880, (2000).

27. http://www.thecatalyst.org/electabl.html, last accessed. 25th March, 2011.

28. D. Harris, Quantitative Chemical Analysis, 7th ed., W. H. Freeman and Company, USA (2007). 